\title{
The future of drug-resistant tuberculosis treatment: learning from the past and the 2019 World Health Organization consolidated guidelines
}

\author{
José A. Caminero ${ }^{1,2}$, Alberto L. García-Basteiro ${ }^{3,4}$, Adrián Rendon ${ }^{5}$, \\ Alberto Piubello ${ }^{2,6}$, Emanuele Pontali ${ }^{7}$ and Giovanni Battista Migliori $\mathbb{0}^{8}$ \\ Affiliations: ${ }^{1}$ Pneumology Dept, General Hospital of Gran Canaria “Dr. Negrin”, Las Palmas de Gran Canaria, \\ Spain. ${ }^{2}$ International Union against Tuberculosis and Lung Disease, Paris, France. ${ }^{3}$ ISGlobal, Hospital Clínic - \\ Universitat de Barcelona, Barcelona, Spain. ${ }^{4}$ Centro de Investigação em Saúde de Manhiça (CISM), Maputo, \\ Mozambique. ${ }^{5}$ Centro de Investigación, Prevención y Tratamiento de Infecciones Respiratorias - CIPTIR, \\ Hospital Universitario de Monterrey, Universidad Autónoma de Nuevo León -UANL, Monterrey, México. \\ ${ }^{6}$ Tuberculosis Division, Damien Foundation, Niamey, Niger. ${ }^{7}$ Dept of Infectious Diseases, Galliera Hospital, \\ Genova, Italy. ${ }^{8}$ Servizio di Epidemiologia Clinica delle Malattie Respiratorie, Istituti Clinici Scientifici Maugeri \\ IRCCS, Tradate, Italy.
}

Correspondence: José A. Caminero, Pneumology Dept, Hospital General de Gran Canaria “Dr. Negrin", Barranco de la Ballena s/n, 35010 Las Palmas de Gran Canaria, Spain. E-mail: jcamlundagobiernodecanarias.org

@ERSpublications

New shorter MDR-TB regimens based on drugs administered orally http://bit.ly/2Nwg6lv

Cite this article as: Caminero JA, García-Basteiro AL, Rendon A, et al. The future of drug-resistant tuberculosis treatment: learning from the past and the 2019 World Health Organization consolidated guidelines. Eur Respir J 2019; 54: 1901272 [https://doi.org/10.1183/13993003.01272-2019].

The World Health Organization (WHO) recently launched the consolidated guidelines on drug-resistant tuberculosis (DR-TB) treatment [1]. They include a new drug classification to manage rifampicin-resistant (RR) and multidrug-resistant (MDR)-TB, while proposing either a shorter regimen (including injectable drugs) or a longer all-oral one as the recommended treatment options. Efficient diagnostic tools are presently available to diagnose resistance to second-line drugs within a few hours (instead of the weeks previously needed) [2]. Therefore, the challenge for National TB Programmes is now to capture these new recommendations in their national guidelines. The aim of this article is to contribute to the ongoing discussion on RR-/MDR-TB treatment considering the lessons learnt over the past 70 years of anti-TB chemotherapy. Other important topics raised in the 2019 WHO drug-resistant treatment guidelines, such as the treatment of isoniazid-resistant $\mathrm{TB}$ or the comparison between the two WHO-approved regimens for MDR-TB, have not been addressed within the constraints of this short editorial.

Anti-TB chemotherapy has dramatically improved TB patients' outcomes. From 1946 to 1976, several anti-TB drugs were discovered, including the two most effective ones, e.g. isoniazid $(\mathrm{H})$ and rifampicin (R). In parallel, randomised controlled trials using several drugs in combination raised the evidence supporting the two core principles of anti-TB chemotherapy: 1) combining at least two drugs (to avoid selection of naturally resistant mutants) for 2) a sufficient duration to cure and prevent relapses (to effectively sterilise the infected tissues) $[3,4]$.

Randomised controlled trials proved that a two-drug regimen was effective in all TB forms if there was no resistance to any of those drugs [3-5]. Based on these findings, since the 1980s a 9-month regimen with 
TABLE 1 Treatment approaches to drug-susceptible (DS) and drug-resistant tuberculosis (TB): number and type of drugs, and treatment duration

\begin{tabular}{|c|c|c|c|c|c|c|}
\hline Reference & $\begin{array}{l}\text { DS-/MDR-/ } \\
\text { XDR-TB }\end{array}$ & $\begin{array}{l}\text { Minimum } \\
\text { number of } \\
\text { drugs in the } \\
\text { regimen }\end{array}$ & $\begin{array}{l}\text { Duration of the } \\
\text { regimen }\end{array}$ & Drugs in the regimen & $\begin{array}{l}\text { Reason for changing lincreasing } \\
\text { or decreasing) the number of } \\
\text { drugs in the regimen }\end{array}$ & $\begin{array}{l}\text { Reason for changing } \\
\text { (increasing or decreasing) } \\
\text { the regimen duration }\end{array}$ \\
\hline $\begin{array}{l}\text { Fox et al. [3] } \\
\text { (1999) } \\
\text { ATS [6] (1986) }\end{array}$ & DS-TB & 2 & 9 months & $H+\mathrm{R}$ & & \\
\hline $\begin{array}{l}\text { Fox et al. [3] } \\
\text { (1999) } \\
\text { ATS [6] (1986) } \\
\text { WHO [7] } \\
\text { (1991) }\end{array}$ & DS-TB & 3 & 6 months & $\mathrm{H}+\mathrm{R}+\mathrm{Z}$ or $2 \mathrm{HRZ} / 4 \mathrm{HR}$ & $\begin{array}{l}\text { Increase: adding } Z \text { to reduce } \\
\text { duration of the treatment course }\end{array}$ & $\begin{array}{l}\text { Decrease: two drugs with } \\
\text { major sterilising activity }\end{array}$ \\
\hline WHO [9] (2003) & DS-TB & 4 & 6 months & 2HRZE/4HR & $\begin{array}{l}\text { Increase: prevent failure in the era } \\
\text { of increasing } H \text { resistance }\end{array}$ & $\begin{array}{l}\text { Decrease: two drugs with } \\
\text { major sterilising activity }\end{array}$ \\
\hline $\begin{array}{l}\text { CAMinero et al. } \\
\text { [4] (2018) }\end{array}$ & DS-TB & $3 / 4$ & 6 months & $\begin{array}{l}\text { 2HRZE/4HR or } 2 \mathrm{HRZ} / 4 \mathrm{HR} \text { if } \\
\text { susceptibility is known before } \\
\text { starting treatment }\end{array}$ & $\begin{array}{l}\text { Increase: prevent failure in the era } \\
\text { of increasing } \mathrm{H} \text { resistance }\end{array}$ & $\begin{array}{l}\text { Decrease: two drugs with } \\
\text { major sterilising activity }\end{array}$ \\
\hline WHO [12] (1996) & MDR-TB & 4 & 21 months & $A G$, Eto, $Z, O f x$ & $\begin{array}{l}\text { Increase: quite a lot of drugs with } \\
\text { very reduced efficacy }\end{array}$ & $\begin{array}{l}\text { Increase: lack of drugs with } \\
\text { strong sterilising activity }\end{array}$ \\
\hline $\begin{array}{l}\text { WHO [13] (2006) } \\
\text { WHO [14] } \\
(2008) \\
\text { WHO [15] } \\
(2011)\end{array}$ & MDR-/XDR-TB & At least 4-5 & $18-24$ months & $\begin{array}{l}\text { Fluoroquinolones (Ofx/Lfx, } \\
\text { moderately sterilising)+others }\end{array}$ & $\begin{array}{l}\text { Increase: several drugs with } \\
\text { limited efficacy }\end{array}$ & $\begin{array}{l}\text { Increase: lack of drugs with } \\
\text { strong sterilising activity }\end{array}$ \\
\hline $\begin{array}{l}\text { WHO [16] (2016) } \\
\text { AHMAD KHAN } \\
\text { et al. [18] } \\
\text { (2017) } \\
\text { NunN et al. } \\
\text { [20] (2019) }\end{array}$ & MDR-TB & 7 & $9-11$ months & Km, Mfx, Pto, Cfz, Z, high-dose H, E & $\begin{array}{l}\text { Increase: to assure bactericidal } \\
\text { activity in the intensive phase }\end{array}$ & $\begin{array}{l}\text { Decrease: two drugs with } \\
\text { major sterilising activity plus } \\
\text { the possible action of } Z \text { if } \\
\text { susceptible }\end{array}$ \\
\hline $\begin{array}{l}\text { AнMAD et al. [21] } \\
\text { (2018) }\end{array}$ & MDR-TB & 5 & $19-22$ months & $\begin{array}{l}\text { All anti-TB drugs, excluding } \mathrm{H} \text { and } \\
\mathrm{R}\end{array}$ & $\begin{array}{l}\text { Increase: several drug with limited } \\
\text { efficacy }\end{array}$ & $\begin{array}{l}\text { Increase: lack of drugs with } \\
\text { strong sterilising activity }\end{array}$ \\
\hline WHO [1] (2019) & MDR-TB & 4 & $\begin{array}{l}\text { Two options: } \\
18-20 \text { months } \\
\text { (including a } 15 \text { to } \\
\text { 17-month } \\
\text { continuation } \\
\text { phase) or } \\
9-11 \text { months }\end{array}$ & $\begin{array}{l}\text { At least four active drugs: always } \\
\text { Lfx/Mfx, Bdq and Lzd+Cfz or } \\
\text { cycloserine/terizidone lother } \\
\text { group C drugs to be employed } \\
\text { when first choices cannot be } \\
\text { used) } \\
\text { Km, high-dose H, Pto, high-dose } \\
\text { Mfx, Cfz, E, Z }\end{array}$ & $\begin{array}{l}\text { Decrease: very active core drugs } \\
\text { Increase: to assure bactericidal } \\
\text { activity in the intensive phase }\end{array}$ & $\begin{array}{l}\text { Increase: not justified because } \\
\text { four sterilising drugs are } \\
\text { included } \\
\text { Decrease: two drugs with } \\
\text { major sterilising activity, } \\
\text { plus the possible action of Z } \\
\text { if susceptible }\end{array}$ \\
\hline $\begin{array}{l}\text { Current paper } \\
2019\end{array}$ & MDR-TB & 3 & 6 & Lfx/Mfx (Cfz), Bdq and Lzd & $\begin{array}{l}\text { Decrease: very active drugs; } \\
\text { unlikely resistance due to } \\
\text { previous exposure }\end{array}$ & $\begin{array}{l}\text { Decrease: three drugs with } \\
\text { major sterilising activity }\end{array}$ \\
\hline
\end{tabular}

ATS: American Thoracic Society; WHO: World Health Organization; MDR: multidrug resistant; XDR: extensively drug resistant; H: isoniazid; R: rifampicin; Z: pyrazinamide; E: ethambutol; AG: aminoglycoside; Eto: ethionamide; Ofx: ofloxacin; Lfx: levofloxacin; Km: kanamycin; Mfx: moxifloxacin; Pto: prothionamide; Cfz: clofazimine; Lzd: linezolid; Bdq: bedaquiline. 
two drugs (isoniazid and rifampicin) or a 6-month regimen with three drugs (HR plus pyrazinamide $(\mathrm{Z})$ ) were recommended by WHO and other major scientific societies (table 1) [3-7]. Rifampicin and pyrazinamide both have high sterilising activity [3-6]; the use of rifampicin allowed decrease of the 1824 month regimens to 9 months, while pyrazinamide further shortened treatment duration to just 6 months $[3,4,6,7]$.

The concept that by using sterilising drugs it is possible to shorten treatment duration was demonstrated in several randomised controlled trials [3-8]. The effectiveness of HR and HRZ regimens became jeopardised when the prevalence of primary isoniazid resistance increased globally, boosting the risk of making HR-based regimens ineffective and creating additional resistances (e.g. transforming an isoniazid-resistant case into a MDR-TB one) [4, 8]. In order to prevent this possibility, WHO recommended addition of a fourth drug, ethambutol $[4,9]$. Its contribution was therefore not necessary if isoniazid resistance was not present [4]. In summary, pyrazinamide and ethambutol were not important for increasing regimen effectiveness, their role being to shorten the regimen (in the case pyrazinamide) and to protect rifampicin in case isoniazid resistance was present (for ethambutol).

Therefore, two highly effective drugs administered for an adequate period of time are sufficient to cure practically all of drug-susceptible cases $[3-8,10]$. However, acquisition of drug resistance is often more complex, especially because it has been proven that drug resistance can develop even if the treatment regimen is correctly prescribed/taken and no resistance is present, due to individual pharmacokinetic variability [11]. In addition, variable drug penetration capacity in lung lesions and variability in strain characteristics of $M$. tuberculosis (i.e. lineage) also play a role in the risk of developing drug resistance.

Since no other drugs with sterilising activity other than rifampicin and pyrazinamide were available for decades, cases of RR- and MDR-TB (or when adverse events to rifampicin occurred) were treated for 18 24 months with less effective drugs, and WHO recommended this from 1996 to 2016 [12-16]. Ofloxacin and levofloxacin ( $\mathrm{Lfx}$ ) were the only fluoroquinolones recommended to treat DR-TB at the time, both with moderate sterilising activity and limited capacity to shorten regimens. Although pyrazinamide was routinely used in MDR-TB cases, it was not considered as a core effective drug because its previous use in almost all cases increased the risk of pyrazinamide resistance.

As new evidence supported a shorter regimen for RR-/MDR-TB [16], WHO recommended its use in 2016 under specific conditions including susceptibility to fluoroquinolones and injectable drugs [16]. This regimen was shortened to 9-11 months [17] because high-dose moxifloxacin ( $\mathrm{Mfx}$ ) and clofazimine (Cfz) have high sterilising activity [4]. The regimen also included pyrazinamide, which could play an important role in treatment shortening in the absence of resistance. A 2017 meta-analysis of studies of this shorter regimen confirmed its effectiveness [18], and which was further demonstrated in nine African countries [19]. In 2019 a non-inferiority randomised controlled trial (the STREAM trial) showed the shorter regimen had similar outcomes to the longer one [20]. It is important to point out that the longer regimen used in the STREAM trial included the "classic" old oral drugs and an injectable agent, very different from the all-oral longer regimen currently recommended by the WHO (which includes new and re-purposed drugs).

In the latest consolidated 2019 MDR-TB guidelines, the all-oral (injectable-free) longer regimen is recommended, while the shorter regimen is still considered a valid option [1]. To design the longer regimen, WHO recommends to combine all three group A drugs (Lfx/Mfx, bedaquiline (Bdq) and linezolid (Lzd)) plus at least one from group B (clofazimine or cycloserine/terizidone (Cs/Tzd)). The total duration of this regimen, which includes at least four drugs, is 18-20 months; 15-17 months of treatment are recommended after achieving bacteriological conversion [1].

The duration of this longer regimen is based on the results of a large individual data meta-analysis [21], which represents the best evidence available to date. In this meta-analysis, a five-drug regimen in the intensive phase and four-drug regimen in the continuation phase was associated with higher treatment success rates (and lower mortality), as compared to 0-2 drug regimens (adjusted odds ratio (aOR) 2.6, 95\% CI 2.1-3.2; and aOR 2.8, 95\% CI 2.2-3.5, respectively). Likewise, better treatment success rates were achieved with regimens lasting 19-22 months [21]. It is noteworthy that this study was based on cohorts treated using several drugs with limited efficacy [21] and poor sterilising activity (ethambutol, prothionamide/ethionamide (Pto/Eto), para-aminosalicylic acid (PAS), Cs/Tzd and injectables) [3, 4, 22, 23]. They were likely to provide a limited contribution to achieve treatment success, as the authors of the study recognise in their conclusions [21]. And, for cycloserine a difference in both mortality and treatment success was found when susceptible and resistant isolates where analysed independently, but not for the remaining drugs (including for ethambutol and pyrazinamide). Fortunately, most of the cohorts included in the meta-analysis included Mfx/Lfx. Table 1 shows the most relevant changes in the number of drugs and treatment duration along with the history of TB and RR-/MDR-TB treatment. 
Considering the principles of anti-TB treatment discussed above, we can argue that a RR-/MDR-TB case sensitive to all the group A drugs could be cured using those three drugs only [10]. And, as all group A drugs have bactericidal and sterilising activity [4, 22-26], a shorter regimen (6-9 months) could possibly cure and prevent relapses [10]. Sensitivity to all group A drugs is a pre-condition to design an effective regimen. Ideally, resistance should be ruled out in all cases. Rapid detection of fluoroquinolone resistance is now available [2], and, as bedaquiline and linezolid have only recently been introduced in anti-TB regimens, primary resistance to them is unlikely (although it is appearing in some settings) [27]. In regions where bedaquiline and linezolid have been introduced recently, ruling out resistance to fluoroquinolones is probably sufficient to use a 6-9 months regimen based on the three group A drugs only. However, although background resistance levels to these compounds (bedaquiline, linezolid and clofazimine) are generally low, this might not be true in all settings and might change rapidly in the future. For this reason, when drug susceptibility testing for bedaquiline and linezolid becomes routinely available, it would ideally be necessary to rule out resistance to these three drugs before starting a regimen which includes them.

On the other hand, if resistance to fluoroquinolones is proven or likely, clofazimine (a bactericidal and sterilising drug from group B) $[4,18,19,22]$, could be a valid alternative. This is clearly not possible for cycloserine, as its bactericidal and sterilising activity is poor $[3,4,22]$. Interestingly, the currently ongoing open-label NIX-TB trial (F. Conradie; unpublished results presented at The Union World Conference on Lung Health, 2018), is based on the same arguments discussed above. It is testing a 6-month, three-drug regimen including linezolid, bedaquiline and pretomanid (which recently started its approval track of the US regulatory authorities) [28]. The NIX-TB trial results will inform future guidelines and clinical practice on all-oral shorter regimens for DR-TB.

Group $\mathrm{C}$ drugs should be used when it is not possible to design an adequate regimen with group A and B drugs. According to WHO, group C drugs are "ranked by decreasing order of usual preference for use", placing ethambutol and pyrazinamide in first and third position, respectively (table 2). It seems the available evidence does not support such order [4, 21, 22]. Actually, in the most recent meta-analysis, ethambutol and pyrazinamide did not show benefits towards treatment success, independently from the susceptible/resistant result of the isolate (aOR 0.9, 95\% CI 0.7-1.1; and 0.7, 95\% CI 0.5-0.9, respectively), while carbapenems (imipenem and meropenem) did (aOR 4.0, 95\% CI 1.7-9.1) [21]. We fear that most clinicians will favour ethambutol or pyrazinamide (more likely to be ineffective for previous use) over better, newer drugs, such as imipenem/meropenem and delamanid, which may be more effective and with a good safety profile $[4,17,29,30]$. Amikacin, although rather toxic, can still be a resource drug in selected cases $[1,21-23]$.

The need for using group $\mathrm{C}$ drugs is justified by the extended resistance pattern of the M. tuberculosis strain. Thus, ideally, we need to prioritise the drugs based on efficacy, in particular looking to their bactericidal and sterilising activity $[4,23,24]$.

Based on 1) the evidence available (from the meta-analysis and other observational studies) $[4,21-24,29,30]$ and 2) the bactericidal/sterilising activity of these drugs, we suggest the following order: imipenem/

\begin{tabular}{|c|c|c|c|c|c|}
\hline \multirow{2}{*}{$\begin{array}{l}2019 \text { World Health Organization } \\
\text { order, group C }\end{array}$} & \multicolumn{2}{|c|}{ Activity } & \multirow{2}{*}{$\begin{array}{l}\text { Proposed } \\
\text { order }\end{array}$} & \multicolumn{2}{|c|}{ Activity } \\
\hline & Bactericidal & Sterilising & & Bactericidal & Sterilising \\
\hline Ethambutol (E) ${ }^{\#}$ & $+/-$ & - & Imp/Mpm & +++ & $+/++?$ \\
\hline Delamanid (DIm) & ++ & ++ & Dlm & ++ & ++ \\
\hline Pyrazinamide $(Z)^{\#}$ & $+/-$ & $++/+++$ & $\mathrm{Am} / \mathrm{S}$ & +++ & - \\
\hline $\begin{array}{l}\text { Imipenem-Cilastatin (Ipm-Cln) or } \\
\text { Meropenem (Mpm) }\end{array}$ & +++ & $+/++$ & Eto/Pto & $+/++$ & - \\
\hline $\begin{array}{l}\text { Amikacin }(\mathrm{Am}) \text { (or } \\
\text { streptomycin (S)) }\end{array}$ & +++ & - & Z & $+/-$ & $++/++$ \\
\hline $\begin{array}{l}\text { Ethionamide (Eto) or } \\
\text { Prothionamide (Pto) }\end{array}$ & $+/++$ & - & $E$ & $+/-$ & - \\
\hline p-aminosalicylic acid (PAS) & - & - & PAS & - & - \\
\hline
\end{tabular}

Based on efficacy, considering both the bactericidal and sterilising activity of the drugs [4, 22]. * : risk of resistance to ethambutol and pyrazinamide is high in patients who have already received them and treatment failed; drug susceptibility testing currently available is not completely reliable for either of these drugs. 
meropenem (despite the relatively small number of patients included in the meta-analysis; the single small "early bactericidal activity" study available and the high operational complexity of administering carbapenems in resource-limited settings), followed by delamanid, amikacin, Eto/Pto, pyrazinamide and ethambutol (table 2).

Moreover, the available evidence does not favour the use of PAS, as the patients exposed to PAS had lower success rate and higher mortality than those not receiving it (aOR 0.8, 95\% CI 0.7-1.0; and 1.2, 95\% CI 1.1-1.4 for treatment success and death, respectively) [21].

Furthermore, Cs/Tzd, currently ranked in group B, could be downgraded in future revisions if we consider their modest bactericidal and sterilising activity $[3,4,8,22]$ and the results of the recent meta-analysis [21]. Lastly, the use of injectables kanamycin and capreomycin is no longer recommended by WHO [1] based on the results of the above-mentioned meta-analysis [21]. However, because of their bactericidal activity $[3-5,22,23]$ they might still represent an option in selected cases when no other drug is available and susceptibility to them has been confirmed.

In conclusion, we believe the recently published WHO guidelines for RR-/MDR-TB treatment represent an important step towards better management of patients with DR-TB.

In this article we provide elements to feed future discussions on the minimum number of drugs and on the duration of regimens to manage RR-/MDR-TB using new drugs. Evidence on the use of new drugs and regimens is rapidly growing [31] and the current concepts and practices will evolve in real time. Quality operational research assessing the benefits of different drug combinations and treatment durations is needed, including shorter regimens (6-9 months) based on Mfx-Bdq-Lzd for RR-/MDR-TB patients with proven fluoroquinolone susceptibility and on Bdq-Lzd-Cfz for those who are resistant to them. While waiting for the results of randomised controlled trials, well-designed operational research projects can provide rapid, and important, answers to improve the quality of MDR-TB care [32].

As far as we know, no trial has been planned yet to study the regimen we are proposing.

The WHO is supportive of testing all-oral regimens, but under operational research conditions only. Additional guidance was recently provided by the Global Drug Initiative document [33], where "modified" shorter regimens are encouraged for fluoroquinolone-susceptible strains only, under operational research conditions and within regimens including four drugs or more.

Acknowledgements: This article belongs to the scientific activities of the WHO Collaborating Centre for Tuberculosis and Lung Diseases, Tradate, ITA-80, 2017-2020- GBM/RC/LDA and the Global Tuberculosis Network (GTN, Committees on treatment, clinical trials and strategies).

Conflict of interest: None declared.

\section{References}

1 World Health Organization. WHO Consolidated Guidelines on Drug-resistant Tuberculosis Treatment. Geneva, World Health Organization, 2019.

2 World Health Organization. The Use of Molecular Line Probe Assays for the Detection of Resistance to Second-line Anti-tuberculosis Drugs. Policy Guidance. Geneva, World Health Organization, 2016. WHO/HTM/ $\mathrm{TB} / 2016.07$.

3 Fox W, Ellard GA, Mitchison DA. Studies on the treatment of tuberculosis undertaken by the British Medical Research Council Tuberculosis Units, 1946-1986, with relevant subsequent publications. Int J Tuberc Lung Dis 1999; 3: Suppl. 2, S231-S279.

4 Caminero JA, Scardigli A, van der Werf TS, et al. Treatment of drug-susceptible and drug-resistant tuberculosis. Eur Respir Monogr 2018: 205-227.

5 Caminero JA, Sotgiu G, Zumla A, et al. Best drug treatment for multidrug-resistant and extensively drug-resistant tuberculosis. Lancet Infect Dis 2010; 10: 621-629.

6 American Thoracic Society and Centers for Disease Control. Treatment of tuberculosis and tuberculosis infection in adults and children. Am Rev Respir Dis 1986; 134: 355-363.

7 World Health Organization. Guidelines for Tuberculosis Treatment in Adults and Children in National Tuberculosis Programmes. Geneva, World Health Organization, 1991. WHO/TUB/91.161.

8 Mitchison DA, Nunn AJ. Influence of initial drug resistance on the response to short-course chemotherapy of pulmonary tuberculosis. Am Rev Respir Dis 1986; 133: 423-430.

9 World Health Organization. Treatment of Tuberculosis: Guidelines for National Programmes. Third Edn. Geneva, World Health Organization, 2003. WHO/CDS/TB/2003.313: 1-108.

10 Caminero JA, García-Basteiro AL, Rendon A. Ideal number of drugs needed and treatment duration to successfully treat multidrug resistant tuberculosis. Lancet 2019; 394: 298.

11 Pasipanodya JG, Mcllieron H, Burger A, et al. Serum drug concentrations predictive of pulmonary tuberculosis outcomes. J Infect Dis 2013; 208: 1464-1473.

12 Crofton J, Chaulet P, Maher D, et al. Guidelines for the Management of Drug-resistant Tuberculosis. Geneva, World Health Organization, 1996.

13 World Health Organization. Guidelines for the Programmatic Management of Drug-resistant Tuberculosis. Geneva, World Health Organization, 2006. WHO/HTM/TB/2006.361. 
14 World Health Organization. Guidelines for the Programmatic Management of Drug-resistant Tuberculosis. Emergency Update 2008. Geneva, World Health Organization, 2008. WHO/HTM/TB/2008.402.

15 World Health Organization. Guidelines for the Programmatic Management of Drug-resistant Tuberculosis. 2011 Update. Geneva, World Health Organization, 2011. WHO/HTM/TB/2011.6.

16 World Health Organization. WHO Treatment Guidelines for Drug-resistant Tuberculosis. 2016 Update. Geneva, World Health Organization, 2016. WHO/HTM/TB/201604. 2016.

17 Aung KJ, Van Deun A, Declercq E, et al. Successful "9-month Bangladesh regimen" for multidrug-resistant tuberculosis among over 500 consecutive patients. Int J Tuberc Lung Dis 2014; 18: 1180-1187.

18 Ahmad Khan F, Salim MAH, du Cros P, et al. Effectiveness and safety of standardised shorter regimens for multidrug-resistant tuberculosis: individual patient data and aggregate data meta-analysis. Eur Respir J 2017; 50: 1700061.

19 Trébucq A, Schwoebel V, Kashongwe Z, et al. Treatment outcome with a short multidrug-resistant tuberculosis regimen in nine African countries. Int J Tuberc Lung Dis 2018; 22: 17-25.

20 Nunn AJ, Philipps PPJ, Meredith SK, et al. A trial of a shorter regimen for rifampin-resistant tuberculosis. $N$ Eng J Med 2019; 380: 1201-1213.

21 The Collaborative Group for the Meta-analysis of Individual Patient Data in MDR-TB Treatment 2017, Ahmad N, Ahuja SD, et al. Treatment correlates of successful outcomes in pulmonary multidrug-resistant tuberculosis: an individual data and meta-analysis. Lancet 2018; 392: 821-834.

22 Caminero JA, Scardigli A. Classification of anti-TB drugs: A new potential proposal based on the most recent evidence. Eur Respir J 2015; 46: 887-893.

23 Caminero J A, Piubello A, Scardigli A, et al. Proposal for a standardised treatment regimen to manage pre- and extensively drug-resistant tuberculosis cases. Eur Respir J 2017; 50: 1700648

24 Tang S, Yao L, Hao X, et al. Efficacy, safety and tolerability of linezolid for the treatment of XDR-TB: a study in China. Eur Respir J 2015; 45: 161-170.

25 Diacon AH, Pym A, Grobusch MP, et al. Multidrug-resistant tuberculosis and culture conversion with bedaquiline. $N$ Engl J Med 2014; 371: 723-732.

26 Rustomjee R, Liehnardt C, Kanyok T, et al. A phase II study of the sterilizing activities of ofloxacin, gatifloxacin and moxifloxacin in pulmonary tuberculosis. Int J Tuberc Lung Dis 2008; 12: 128-138.

27 Veziris N, Bernard C, Guglielmetti L, et al. Rapid emergence of Mycobacterium tuberculosis bedaquiline resistance: lessons to avoid repeating past errors. Eur Respir J 2017; 49: 1601719.

28 TB Alliance. FDA Advisory Committee Votes Favorably on the Question of the Effectiveness and Safety of Pretomanid in Combination with Bedaquiline and Linezolid for Treatment of Highly Drug-Resistant Forms of Tuberculosis. June 6, 2019. www.tballiance.org/news/fda-advisory-committee-votes-favorably-question-effectivenessand-safety-pretomanid-combination. Date last updated: 6 June 2019. Date last accessed: 7 June 2019.

29 Tiberi S, Sotgiu G, D'Ambrosio L, et al. Comparison of effectiveness and safety of imipenem/clavulanate- versus meropenem/clavulanate-containing regimens in the treatment of MDR- and XDR-TB. Eur Respir J 2016; 47: $1758-1766$.

30 Pontali E, Sotgiu G, Tiberi S, et al. Combined treatment of drug-resistant tuberculosis with bedaquiline and delamanid: a systematic review. Eur Respir J 2018; 52: 1800934

31 Pontali E, Raviglione MC, Migliori GB, et al. Regimens to treat multidrug-resistant tuberculosis: past, present and future perspectives. Eur Respir Rev 2019; 28: 190035.

32 Sotgiu G, Centis R, D'Ambrosio L, et al. Linezolid to treat extensively drug-resistant TB: retrospective data are confirmed by experimental evidence. Eur Respir J 2013; 42: 288-290.

33 Global Drug-resistant TB Initiative, Stop TB Partnership. The Evaluation of Effectiveness and Safety of Novel Shorter Treatment Regimens for Multidrug-Resistant Tuberculosis. 2018. Available from http://www.stoptb.org/wg/ mdrtb/assets/documents/GDI\%20OR\%20generic\%20protocol\%20final.pdf 